\title{
Radiological patterns of secondary sclerosing cholangitis in patients after lung transplantation
}

\author{
Hye Jin Kim, ${ }^{1,3}$ Mi-Suk Park $\odot,{ }^{1,2}$ Jin Woo Son, ${ }^{1}$ Kyunghwa Han, ${ }^{2}$ Jei Hee Lee, ${ }^{3}$ \\ Jai Keun Kim, ${ }^{3}$ and Hyo Chae Paik ${ }^{4}$
}

${ }^{1}$ Department of Radiology, Severance Hospital, Yonsei Biomedical Research Institute, Yonsei University College of Medicine, 50-1 Yonsei-Ro, Seodaemun-Gu, Seoul 03722, Korea

${ }^{2}$ Department of Radiology and Research Institute of Radiological Science, Severance Hospital, Yonsei University College of Medicine, 50-1 Yonsei-Ro, Seodaemun-Gu, Seoul 03722, Korea

${ }^{3}$ Present address: Department of Radiology, Ajou University School of Medicine, 164 Worldcup-ro, Yeongtong-gu, Suwon 16499, Korea

${ }^{4}$ Department of Thoracic and Cardiovascular Surgery, Severance Hospital, Yonsei University College of Medicine, 50-1 Yonsei-Ro, Seodaemun-Gu, Seoul 03722, Korea

\section{Abstract}

Purpose: The purpose of this study was to investigate the radiological patterns of secondary sclerosing cholangitis (SSC) following lung transplantation.

Methods: Fifty-five patients underwent abdominopelvic CT before and after lung transplantation for end stage pulmonary disease. Nine patients underwent MR cholangiopancreatography (MRCP). The radiological patterns of biliary abnormalities (location, bile duct dilatation with stricture, beaded appearance, and biliary casts/ sludge), laboratory data (serum total bilirubin and alkaline phosphatase), and patient survival rates were evaluated. SSC was diagnosed when there were newly developed biliary abnormalities with cholestasis after lung transplantation. Potential perioperative risk factors pertaining to SSC were analyzed. Patient survival rates with or without SSC were compared.

Results: Six of the 55 patients showed imaging and laboratory findings of SSC after lung transplantation. Multifocal dilatation and stricture involved the intrahepatic (6 of $6,100 \%$ ), hilar (4 of $6,66.7 \%$ ), and extrahepatic bile duct ( 1 of $6,16.7 \%)$. On CT, the lesions presented as multifocal small cyst-like lesions along the bile duct course. On MRCP, the lesions showed beaded appearance with mild duct dilatation. Preoperative mechanical ventilation and bilateral lung transplantation were associated with $\operatorname{SSC}(p<0.05)$. The median

Electronic supplementary material The online version of this article (https://doi.org/10.1007/s00261-018-1819-2) contains supplementary material, which is available to authorized users. survival of patients with SSC was 4.6 months.

Conclusion: Lung transplantation can induce SSC similar to SSC in critically ill patients, and result in worse clinical outcomes than in patients without SSC. Multifocal small cyst-like lesions along the intrahepatic bile duct on CT and beaded appearance on MRCP are suggestive findings of SSC in patients with cholestasis after lung transplantation.

Key words: Secondary sclerosing cholangitis-Lung transplantation-CT-MRCP

Secondary sclerosing cholangitis (SSC) is a number of variably progressive cholestatic disease of the bile duct with identified causes, unlike primary sclerosing cholangitis (PSC) of idiopathic cause [1-3]. A new sub-entity of secondary sclerosing cholangitis has been defined in critically ill patients (SSC-CIP) in recent years [4-9]. This type of SSC occurs in association with intensive-care treatment of patients with major surgery, trauma, burns, and other life-threatening events [4-9]. The exact pathological mechanisms of SSC-CIP have not been elucidated conclusively, but are most likely caused by severe arterial hypotension or shock [4-9]. SSC-CIP is a progressive cholestatic liver disease characterized by necrosis of the bile duct epithelium, which leads to the rapid destruction of the bile ducts [4-9]. SSC-CIP has an irreversible course and less favorable prognosis than SSC of other causes such as drug, infection, or autoimmune diseases [4-9].

Correspondence to: Mi-Suk Park; email: RADPMS@yuhs.ac 
Lung transplantation is the best treatment for patients with end stage pulmonary disease, and the number of lung transplantations has steadily increased [10-13]. As lung transplantation has become more widespread, several complications after lung transplantation have been reported [10-13]. Recently, we have encountered cases of SSC after lung transplants in our center. To our knowledge, however, the radiological findings of SSC in patients with lung transplants have never been reported. The aim of our study was to report the radiological patterns of SSC in patients after lung transplantation.

\section{Materials and methods}

This case series was approved by our institutional review board and the requirement for informed consent from patients was waived.

\section{Patients}

A total of 101 consecutive patients underwent lung transplantation for end-stage pulmonary disease between November 2012 and December 2016 at a single institution. Fifty-five patients underwent abdominopelvic CT before and after lung transplantation $(n=55)$. Nine patients underwent MR cholangiopancreatography (MRCP). Detailed baseline demographic and clinical characteristics of the patients are shown in Table 1.

Table 1. Baseline characteristics of patients with abdominopelvic CT after lung transplantation

\begin{tabular}{ll}
\hline Patient characteristics & $\begin{array}{c}\text { Total patients } \\
(n=55)\end{array}$ \\
\hline Age, year & $52.6 \pm 13.4$ \\
Male gender & $30(54.5)$ \\
Lung transplantation type, bilateral & $11(20)$ \\
Ever smoker & $24(43.6)$ \\
Primary lung disease & $55(100)$ \\
Idiopathic pulmonary fibrosis & $30(54.5)$ \\
Graft versus host disease after PBSCT & $7(12.7)$ \\
Interstitial lung disease associated with & $6(10.9)$ \\
CTD & \\
Lymphangioleiomyomatosis & $3(5.5)$ \\
Idiopathic pulmonary artery hypertension & $2(3.6)$ \\
Others & $7(12.7)$ \\
Previous liver disease & $11(20.0)$ \\
Hepatitis B virus carrier & $6(54.5)$ \\
Alcoholic liver disease & $3(27.3)$ \\
Cardiac cirrhosis & $1(9.1)$ \\
Hepatitis A virus infection & $1(9.1)$ \\
Causes resulting in abdominopelvic CT & $55(100)$ \\
Abdominal pain or distension & $19(34.5)$ \\
Abnormal liver enzyme & $9(16.4)$ \\
Gastrointestinal tract bleeding & $6(10.9)$ \\
Sepsis & $5(9.1)$ \\
Underlying liver disease evaluation & $5(9.1)$ \\
Free air at simple abdominal X-ray & $3(5.5)$ \\
Others & $8(14.5)$ \\
\hline
\end{tabular}

Data are number $(\%)$ of patients or mean $\pm \mathrm{SD}$

PBSCT peripheral blood stem cell transplantation, CTD connective tissue disease

\section{Biliary imaging analysis}

We identified SSC after lung transplantation when all of the following conditions were met: (1) No bile duct dilatation or abnormality on CT imaging before lung transplantation; (2) newly developed intra- or extrahepatic bile duct dilatation and stricture on CT or MRCP after lung transplantation; and (3) elevated serum total bilirubin $(>1 \mathrm{mg} / \mathrm{dL})$ and serum alkaline phosphatase $(>150 \mathrm{U} / \mathrm{L}$ ) levels reflecting cholestasis.

Two board-certified abdominal radiologists with 13 and 17 years of experience in abdominal imaging retrospectively reviewed each pair of abdominopelvic CT images obtained before and after lung transplantation in consensus and analyzed the presence of biliary abnormality. If biliary abnormality was present, the following imaging features were recorded: extent and location (intrahepatic, hepatic hilum, extrahepatic), degree (mild, moderate, or severe) of dilatation and stricture, presence of beaded appearance, presence of bile duct wall thickening, and presence and location of biliary stones or casts. The presence or absence of acute cholecystitis was also evaluated. Both reviewers were aware that all patients had undergone lung transplantation, but blinded to the other clinical information, laboratory findings, and patient survival.

\section{Clinical findings and patient survival}

Clinical data before, during, and after transplantation were collected from electronic medical records by the third radiologist. The use of mechanical ventilator or extracorporeal membrane oxygenation (ECMO) was investigated before transplantation. The total ECMO time and hypovolemic event during transplantation were also investigated. Cytomegalovirus (CMV) infection, rejection, sepsis, hypovolemia due to bleeding, and other clinically threatened diseases occurring after transplantation were investigated. Serum total bilirubin, alkaline phosphatase levels, time to SSC, and the mean follow-up period were recorded. Patient survival rates after lung transplantation were calculated based on the date of lung transplantation until death or the date of last follow-up evaluation. Censoring was done at the last follow-up date for patients who were lost to follow-up.

\section{$C T$ and MR imaging protocol}

All abdominopelvic CT images were obtained with a 64-channel CT scanner (Sensation 64, Siemens Medical Solutions, Forchheim, Germany; Brilliance 64, Philips Healthcare, Cleveland, OH, USA). After performing non-contrast CT, contrast-enhanced images were obtained $55 \mathrm{~s}$ after reaching $100 \mathrm{HU}$ attenuation in the abdominal aorta with bolus tracking after intravenous administration of non-ionic contrast medium (Ultravist; 
iopromide, Beyer Healthcare, Berlin, Germany and Omnipaque; iohexol, GE Healthcare, Cork, Ireland). The scanning parameters were as follows: beam collimation, $0.625 \mathrm{~mm}$; slice thickness, $3 \mathrm{~mm}$; reconstruction interval, $3 \mathrm{~mm}$; pitch, 0.6 ; rotation time, $0.5 \mathrm{~s}$; effective tube current-time charge, $170-240 \mathrm{mAs} ; 100-120 \mathrm{kVp}$.

Nine of 55 patients underwent MRCP with a 3.0-T scanner (Magnetom Trio Tim; Siemens Medical Solutions, Erlangen, Germany), using a 16-channel torsoarray coil. Both single-section and navigator-triggered MRCP imaging were performed. Detailed MR imaging parameters are provided in Online Resource 1.

\section{Statistical analysis}

Clinical characteristics of the patients were compared between the SSC and non-SSC groups. The independent two-sample $t$ test or Mann-Whitney $U$ test was used for continuous variables, and the Chi-square test or Fisher's exact test was used for categorical variables. Patient survival was compared between the two groups by using Kaplan-Meier survival analysis and log-rank tests. All statistical analyses were performed using $\mathrm{R}$ software (version 3.3.1.; R Foundation for Statistical Computing, Vienna, Austria). All reported $p$ values were two-sided with a significance level of $<0.05$.

\section{Results}

Six of the 55 patients showed imaging and laboratory findings of SSC after lung transplantation (Figs. 1 and 2). Multifocal dilatation and stricture involve the intrahepatic duct from second order biliary radical to peripheral duct ( 6 of $6,100 \%)$, hilar duct (4 of $6,66.7 \%$ ), and extrahepatic bile duct (1 of 6,16.7\%). On CT, the lesions presented as multifocal small cyst-like lesions along the course of the bile duct. On MRCP, the lesions showed beaded appearance with mild-degree duct dilatation. No patient exhibited bile duct wall thickening, biliary cast, or intrahepatic duct stones. Four patients had gallbladder stones without acute cholecystitis. The serum mean total bilirubin and alkaline phosphatase levels in 6 patients with $\mathrm{SSC}$ was $9.9 \mathrm{mg} / \mathrm{dL}$ (median, 4.3; range, $1.9-26.4 \mathrm{mg} / \mathrm{dL}$ ) and $812 \mathrm{U} / \mathrm{L}$ (median, 732.5; range, 509-1274 U/L), respectively. The mean time to SSC after lung transplantation was 71 days (median, 70 days; range, 53-90 days).

The clinical factors associated with SSC were application of mechanical ventilation before lung transplantation and bilateral type lung transplantation (Table 2).

The mean follow-up period in 55 patients was 22.6 months (median, 16.9 months; range, 1.1-61.8 months). Overall survival rates 90 days, 180 days, and at last follow-up after lung transplantation in 49 patients without SSC were 90.8, 64.9, and 46.3\%, respectively, whereas survival rates in 6 patients with
SSC were 100,100 , and $0 \%$, respectively $(p=0.0008)$ (Table 3 and Fig. 3). The median survival of patients with SSC was 4.6 months.

\section{Discussion}

Our cases of SSC in patients with lung transplantation showed mild intrahepatic bile duct dilatation with multifocal short segmental strictures. On CT, the lesions presented as multifocal small cyst-like lesions along the intrahepatic bile duct course. On MRCP, the lesions showed beaded appearance. All patients involved the peripheral intrahepatic duct. Only one patient had both intra- and extrahepatic duct abnormalities, and no patient had extrahepatic duct abnormalities only.

Unlike our cases, in most patients with liver transplantation SSC primarily involves the extrahepatic bile duct or hepatic hilar duct [14-17]. Blood supply to the bile duct comes solely from the peribiliary plexus of the terminal hepatic artery, which forms a rich microvascular network surrounding the bile ducts. Meanwhile, the majority of the blood supply from the hepatic artery is supplied by retroduodenal or retroportal arteries, which are responsible for the lower port of the bile duct [14, 18]. Therefore, the extrahepatic bile duct, especially the middle third of the common bile duct, is more vulnerable to biliary ischemia or necrosis rather than the intrahepatic bile duct. On the other hand, SSC-CIP is another cause of ischemic cholangiopathy, which is one of the subcategories causing SSC. Hemodynamic instability, mechanical ventilation, required vasopressor support, infection, or prone position in patients receiving intensive care treatment for a variety of life-threatening events have been known to be contributing factors to SSC-CIP $[1,14,18]$. These factors are thought to cause diffuse bile duct ischemia or necrosis more commonly in the intrahepatic bile duct rather than in the extrahepatic bile duct due to subacute to chronic state of sustained insufficient blood supply to the bile duct during a certain period [18]. Therefore, it may show a different pattern from the bile duct ischemia or necrosis due to acute bleeding events that often occur in patients after liver transplantation.

The radiological findings of our cases are similar to those of SSC in critically ill patients [4, 6, 7]. Abnormal bile duct conditions involved the intrahepatic bile duct in more than $87 \%$ of patients, whereas extrahepatic duct changes only were observed in less than $6 \%[4,6]$. Irregular intrahepatic bile ducts with multiple strictures and dilatations were observed on endoscopic retrograde cholangiopancreatography (ERCP), which is similar to PSC [4, 6, 7]. On ERCP, however, enhancement or thickening of the bile duct wall could not be evaluated. Our study using CT and MRCP showed no enhancement or thickening of the bile duct wall, which is different from PSC. Because there was no enhancement or thickening of the ductal wall in our study, the lesions looked 


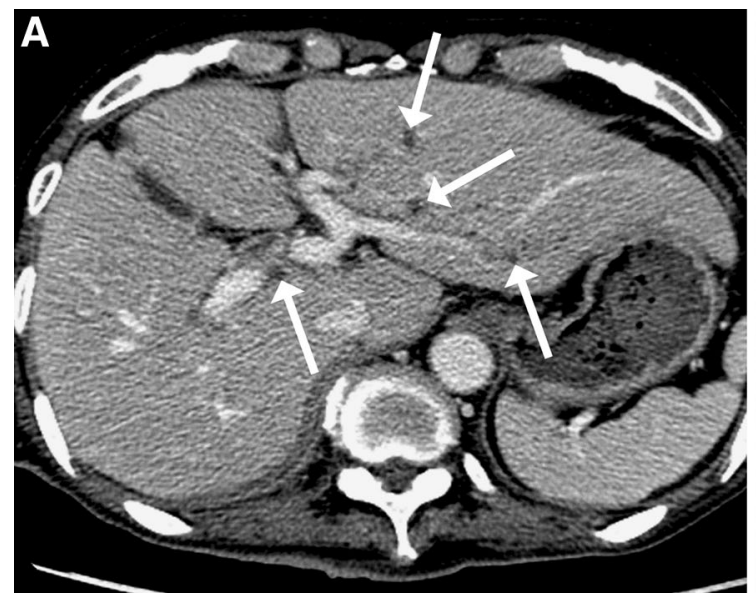

Fig. 1. A 66-year-old man who underwent bilateral lung transplantation for idiopathic pulmonary fibrosis. A CT image obtained 56 days after lung transplantation shows multifocal small cystic lesions (arrows) along the expected bilateral intrahepatic bile duct course, suggesting IHD dilatation. Bile

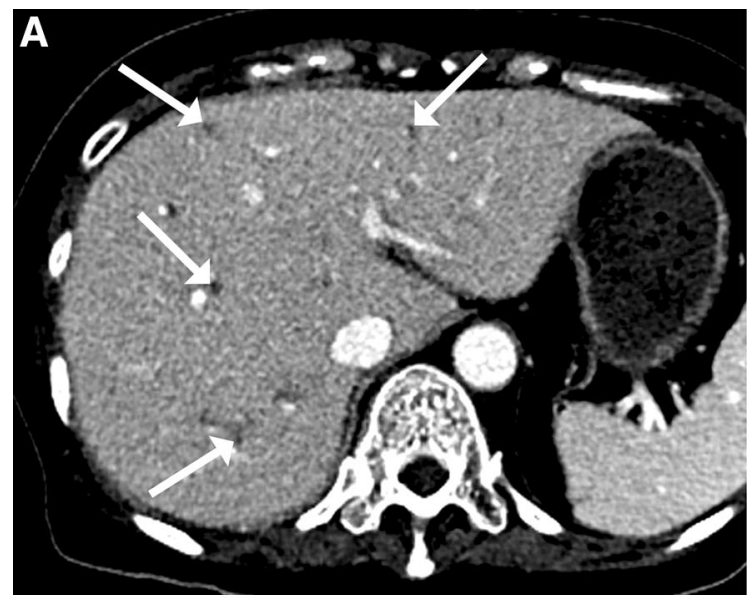

Fig. 2. A 54-year-old woman who underwent bilateral lung transplantation for bronchiolitis obliterans. A CT obtained 63 days after lung transplantation shows multifocal mild intrahepatic ductal dilatation (arrows). Bile duct wall thickening is not observed. B Two-dimensional MRCP

like small cysts along the bile duct on cross-sectional CT. On projectional MRCP images, the bile duct lesions showed beaded appearance similar to PSC.

Leonhardt et al. [4] reported three different cholangiographic features of SSC-CIP corresponding to the stages of pathogenesis: (I) early stage; evidence of biliary casts, (II) intermediate stage; progressive destruction of intrahepatic bile ducts, and (III) late stage; obliteration of the intrahepatic bile ducts/picture of the pruned tree. They suggested that as bile duct destruction progressed, from peripheral to the central duct, the pruned tree appearance would appear in the late stage. ERCP has been recommended for the diagnosis of SSC-CIP [4]. ERCP, however, is not only an invasive procedure but

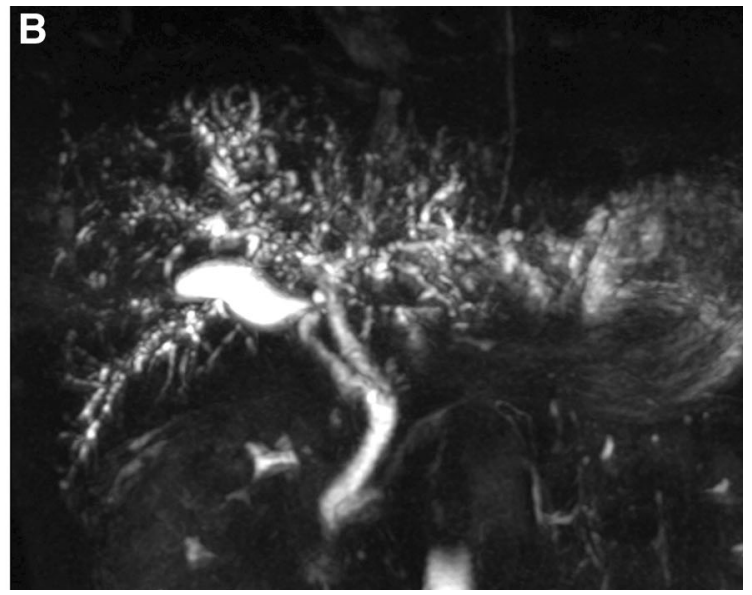

duct wall thickening is not observed. B Two-dimensional MRCP performed 1 day after CT reveals mild multifocal intrahepatic duct dilatation with stricture from hepatic hilum to peripheral duct. Note that extrahepatic bile duct is normal in appearance.

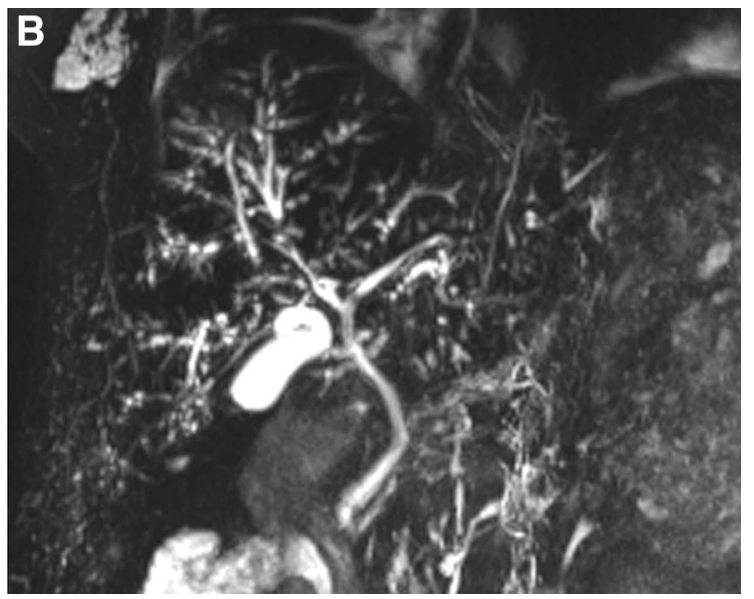

performed 7 days after CT reveals multiple intrahepatic ductal dilatation with stricture from second order biliary radical to peripheral duct. Note that hepatic hilum and extrahepatic bile duct shows normal appearance and that involved intrahepatic bile ducts show multi-cystic appearance.

also plays a limited role in evaluating the intrahepatic bile duct distal to the obstruction. In our study, MRCP without contrast enhancement resulted in typical cholangiographic findings similar to those on ERCP and visualized all of the intrahepatic bile duct. Therefore, MRCP could replace ERCP for the diagnosis of SSCCIP.

Critically ill patients encompass a wide range of patients with life-threatening conditions such as major cardiothoracic surgery, trauma, burns, or sepsis [5, 8, 9]. Because lung transplantation is major surgery requiring the intensive care unit treatment during the perioperative period, it is plausible that lung transplantation is associated with SSC-CIP. The number of lung transplants 
Table 2. Comparison of perioperative and postoperative risk factors between patients with and without secondary sclerosing cholangitis

\begin{tabular}{|c|c|c|c|}
\hline Factors & $\begin{array}{l}\text { Patients with secondary } \\
\text { sclerosing cholangitis }(n=6)\end{array}$ & $\begin{array}{l}\text { Patients without secondary } \\
\text { sclerosing cholangitis }(n=49)\end{array}$ & $p$ \\
\hline \multicolumn{4}{|l|}{ Recipient } \\
\hline Age & $58.8 \pm 6.2(61 ; 49-66)$ & $51.8 \pm 13.9(55 ; 17-71)$ & $0.3045^{\mathrm{a}}$ \\
\hline Sex, male & $4(66.7 \%)$ & $26(53.1 \%)$ & $0.6779^{\mathrm{b}}$ \\
\hline Ever Smoker & $2(33.3 \%)$ & $22(44.9 \%)$ & $0.6862^{\mathrm{b}}$ \\
\hline Previous liver disease & $2(33.3 \%)$ & $7(14.3 \%)$ & $0.2513^{\mathrm{b}}$ \\
\hline \multicolumn{4}{|l|}{ Perioperative factors } \\
\hline Lung transplantation, bilateral & $6(100 \%)$ & $5(10.2 \%)$ & $<0.0001^{\mathrm{b}}$ \\
\hline Pre-mechanical ventilation & $5(83.3 \%)$ & $8(16.3 \%)$ & $0.0019^{\mathrm{b}}$ \\
\hline Pre-ECMO & $2(33.3 \%)$ & $8(16.3 \%)$ & $0.2976^{\mathrm{b}}$ \\
\hline Total ECMO time & $10395.7 \pm 16311.5(614 ; 308-38858)$ & $3357.4 \pm 5823.2(420 ; 160-23,860)$ & $0.2457^{\mathrm{a}}$ \\
\hline \multicolumn{4}{|l|}{ Postoperative factors } \\
\hline Cytomegalovirus infection & $5(83.3 \%)$ & $41(83.7 \%)$ & $>0.9999^{\mathrm{b}}$ \\
\hline Hypovolemic event & $0(0 \%)$ & $7(14.3 \%)$ & $>0.9999^{\mathrm{b}}$ \\
\hline Sepsis & $4(66.7 \%)$ & $19(38.8 \%)$ & $0.2234^{\mathrm{b}}$ \\
\hline Rejection & $0(0 \%)$ & $7(14.3 \%)$ & $>0.9999^{\mathrm{b}}$ \\
\hline \multicolumn{4}{|c|}{ Other factors associated with elevated total bilirubin } \\
\hline HLH & $1(100 \%)$ & $1(100 \%)$ & - \\
\hline Bleeding & $0(100 \%)$ & $2(100 \%)$ & - \\
\hline Thrombotic microangiopathy & $0(100 \%)$ & $4(100 \%)$ & - \\
\hline
\end{tabular}

ECMO extracorporeal membranous oxygenation, $H L H$ hemophagocytic lymphohistiocytosis

${ }^{a}$ Continuous values are mean $\pm \mathrm{SD}$ with median and minimum to maximum in parentheses

${ }^{\mathrm{b}}$ Categorical values are numbers with percentages in parentheses

Table 3. Survival rates (\%) in patients with lung transplantation

\begin{tabular}{|c|c|c|c|}
\hline $\begin{array}{l}\text { Time after lung } \\
\text { transplantation }\end{array}$ & $\begin{array}{l}\text { Patients with secondary } \\
\text { sclerosing cholangitis }(n=6)\end{array}$ & $\begin{array}{l}\text { Patients without secondary } \\
\text { sclerosing cholangitis }(n=49)\end{array}$ & $p$ \\
\hline 30 Days survival & 100 & 100 & - \\
\hline 90 Days survival & 100 & 89.7 & - \\
\hline Last survival & 0 & 52.2 & 0.0008 \\
\hline
\end{tabular}

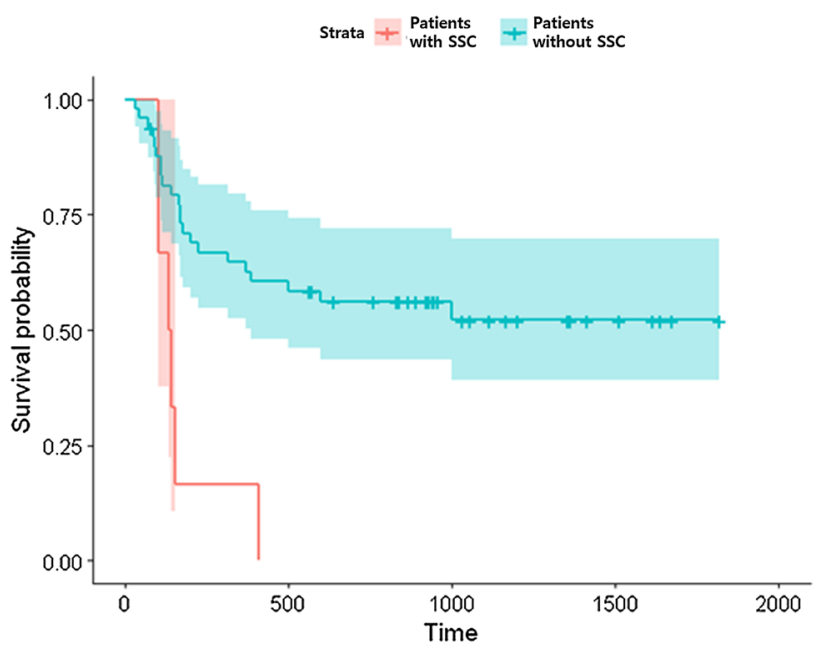

Fig. 3. Kaplan-Meier survival analysis of patients with secondary sclerosing cholangitis (SSC) and without SSC after lung transplantation. Patients with SSC showed that they had a significantly lower survival rate than those without SSC $(p=0.0008)$. has steadily increased worldwide, although patient survival rates after lung transplantation are consistently lower than those of other solid organ transplantations. The mean overall survival of patients with SSC after lung transplantation in our study was only 4.6 months. We found that preoperative mechanical ventilation was associated with SSC after lung transplantation. This result was consistent with previous reports $[1,5]$. However, other known risk factors such as transplant rejection or CMV infection were not associated with SSC in this study. CMV is one of the most frequently infected viruses after lung transplantation and its related vasculitis were known as inducing ischemic cholangiopathy [11, 14]. With regard to sepsis as a possible cause of ischemic cholangiopathy, there was no association between patients with and without SSC. Further studies to identify risk factors of SSC after lung transplantation are needed.

Our study has inevitable limitations related to its retrospective study design and small number of cases. Thus, the findings of SSC after lung transplantation detected in our study cannot be generalized. Lung transplantation is not a widely performed operation, but the 
identification and treatment of SSC after lung transplantation is critical. Therefore, clinicians and radiologists should be aware of the clinical significance and be familiar with the radiological findings of SSC after lung transplantation.

In conclusion, lung transplantation can induce SSC, similar to SSC in critically ill patients, and result in worse clinical outcomes compared to those of patients without SSC. Multifocal small cyst-like lesions along the intrahepatic bile duct on CT and beaded appearance on MRCP are suggestive findings of SSC in patients with cholestasis after lung transplantation.

\section{Compliance with ethical standards}

Funding No funding was received for this retrospective study.

Conflict of interest The authors declare that they have no conflict of interest.

Disclosures of potential conflicts of interest There is no any direct or potential financial disclosure for each author.

Ethical approval All procedures performed in studies involving human participants were in accordance with the ethical standards of the institutional and/or national research committee and with the 1964 Helsinki declaration and its later amendments or comparable ethical standards. For this type of study formal consent is not required.

Informed consent Need of informed consent was waived since this study was performed retrospectively.

\section{References}

1. Brooling J, Leal R (2017) Secondary sclerosing cholangitis: a review of recent literature. Curr Gastroenterol Rep 19:44

2. Seo N, Kim SY, Lee SS, et al. (2016) Sclerosing cholangitis: clinicopathologic features, imaging spectrum, and systemic approach to differential diagnosis. Korean J Radiol 17:25-38

3. Imam MH, Talwalkar JA, Lindor KD (2013) Secondary sclerosing cholangitis: pathogenesis, diagnosis, and management. Clin Liver Dis 17:269-277
4. Leonhardt S, Veltzke-Schlieker W, Adler A, et al. (2015) Secondary sclerosing cholangitis in critically ill patients: clinical presentation, cholangiographic features, natural history, and outcome: a series of 16 cases. Medicine 94:e2188

5. Lin T, Qu K, Xu X, et al. (2014) Sclerosing cholangitis in critically ill patients: an important and easily ignored problem based on a German experience. Front Med 8:118-126

6. Voigtländer T, Negm A, Schneider A, et al. (2012) Secondary sclerosing cholangitis in critically ill patients: model of end-stage liver disease score and renal function predict outcome. Endoscopy 44:1055-1058

7. Gelbmann CM, Rummele P, Wimmer M, et al. (2007) Ischemic-like cholangiopathy with secondary sclerosing cholangitis in critically ill patients. Am J Gastroenterol 102:1221-1229

8. Ben-Ari Z, Levingston D, Weitzman E, et al. (2015) Secondary sclerosing cholangitis following major burn. Ann Hepatol 14:695-701

9. Engler S, Elsing C, Flechtenmacher C, et al. (2003) Progressive sclerosing cholangitis after septic shock: a new variant of vanishing bile duct disorders. Gut 52:688-693

10. Sabashnikov A, Weymann A, Mohite PN, et al. (2014) Risk factors predictive of one-year mortality after lung transplantation. Eur $\mathbf{J}$ Cardiothorac Surg 46:e82-e88

11. Grimm JC, Valero V 3rd, Magruder JT, et al. (2015) A novel risk score that incorporates recipient and donor variables to predict 1-year mortality in the current era of lung transplantation. J Heart Lung Transplant 34:1449-1454

12. Lee SH, Park MS, Song JH, et al. (2017) Perioperative factors associated with 1-year mortality after lung transplantation: a singlecenter experience in Korea. J Thoracic Dis 9:4006-4016

13. Lee SH, Park MS, Lee JG, et al. (2017) Rare causes of hyperbilirubinemia after lung transplantation: our experience at a single center. J Thoracic Dis 9:5030-5039

14. Deltenre P, Valla DC (2008) Ischemic cholangiopathy. Semin Liver Ds $28: 235-246$

15. Mourad MM, Algarni A, Liossis C, Bramhall SR (2014) Aetiology and risk factors of ischaemic cholangiopathy after liver transplantation. WJG 20:6159-6169

16. Kochhar G, Parungao JM, Hanouneh IA, Parsi MA (2013) Biliary complications following liver transplantation. WJG 19:2841-2846

17. Novellas S, Caramella T, Fournol M, Gugenheim J, Chevallier P (2008) MR cholangiopancreatography features of the biliary tree after liver transplantation. AJR 191:221-227

18. Batts KP (1998) Ischemic cholangitis. Mayo Clin Proc 73:380-385 